\title{
SnRNP Biogenesis
}

National Cancer Institute

\section{Source}

National Cancer Institute. snRNP Biogenesis. NCI Thesaurus. Code C30092.

The 4 major small nuclear ribonucleoprotein particles (snRNPs), U1, U2, U4/U6, and U5, share 8 proteins which form the snRNP structural core: SNRPB, SNRPD1, SNRPD2, SNRPD3, SNRPE, SNRPF, and SNRPG. These common proteins play an essential role in the biogenesis of the snRNPs. The assembly of the common proteins onto the small nuclear RNA (snRNA) appears to occur in at least 2 steps and involves the formation of RNA-free protein heterooligomers. (from OMIM) 